COMECHINGONIA. REVISTA DE ARQUEOLOGÍA

Número 16, primer semestre de 2012, pp. 53-70, Córdoba

ISSN 0326-7911

\title{
LA MUJER EN EL REGISTRO BIOARQUEOLOGICO Y SU VISIBILIDAD EN LOS CONTEXTOS FUNERARIOS
}

\author{
E. Inés Baffi ${ }^{1}$ y Verónica Seldes ${ }^{2}$ \\ ${ }^{1}$ Museo Etnográfico J.B. Ambrosetti - Facultad de Filosofía y Letras UBA- CONICET, E-mail: einesbaffi@yahoo.es \\ ${ }^{2}$ INAPL - CONICET- Facultad de Filosofía y Letras UBA
}

Presentado el: 29/11/2011 - Aceptado 01/03/2012

\begin{abstract}
Resumen
En este trabajo nos proponemos realizar una serie de reflexiones sobre el tratamiento dado al mundo femenino en el noroeste argentino a partir de su representación en el registro bioarqueológico y en los contextos funerarios. Se considerará el desarrollo que esta problemática tuvo en la historia de la antropología biológica, en especial la bioarqueología, focalizando en las representaciones de lo femenino en los rituales funerarios y en los diferentes estilos de vida de los pueblos prehispánicos. Se realiza un relevamiento de la información disponible sobre el papel de la mujer en las sociedades prehispánicas a través del análisis de los patrones de residencia, estado de salud y enfermedad, patologías asociadas al sexo y participación de la mujer en situaciones de violencia interpersonal. Se discutirá sobre los distintos enfoques en el análisis bioarqueológico, que permiten evidenciar estos patrones de comportamiento a la vez que deconstruir los lugares tradicionalmente otorgados desde la arqueología y la antropología biológica a la mujer en la organización social de los pueblos prehispánicos. Se tomarán como referencia casos del valle Calchaquí (pcia. de Salta) y quebrada de Humahuaca (pcia. de Jujuy) los cuales permiten dar cuenta de la problemática de la visibilidad femenina.
\end{abstract}

Palabras claves: Noroeste argentino, Antropología biológica, Género, Mujer.

\begin{abstract}
In this paper we propose a series of reflections on the treatment given to the world of women in northwestern Argentina from its representation in the bioarchaeological record and funerary contexts. Development is considered that this issue had in the history of biological anthropology, especially bioarchaeology, focusing on representations of the feminine in the funeral rites and the different lifestyles of the peoples of prehispanic northwestern Argentina. We performed a survey of available information on the role of women in prehispanic societies through the analysis of patterns of residence, health status and disease pathologies associated with gender and participation of women in situations of interpersonal violence. We will discuss different approaches in the bioarchaeological analysis, that reveal the patterns of behavior while deconstructing the places traditionally granted from archeology and biological anthropology to women in the social organization of prehistoric peoples. Cases of the Calchaqui Valley (province of Salta) and Quebrada de Humahuaca (province of Jujuy) will be taken as a reference to account the problem of women's visibility.
\end{abstract}

Keywords: Argentine Northwest, Biological anthropology, Gender, Women. 


\section{Introducción}

Este trabajo se propone focalizar en la problemática de la mujer y su visibilidad en las investigaciones arqueológicas del noroeste argentino. Para esto se realiza un breve recorrido por los trabajos publicados a la vez que se reflexiona sobre el tratamiento históricamente dado por la antropología biológica y la bioarqueología a estas temáticas.

La antropología biológica y dentro de ella, la bioarqueología, ha recorrido distintas perspectivas acorde a los momentos históricos transitados por la ciencia en nuestro país. En su desarrollo y respondiendo a los marcos teóricos y paradigmas que la acompañaron y le dieron sustento (Baffi 2009, Carnese et al. 1997, Seldes 2004), se visualiza la importancia que se le fue otorgando a distintas problemáticas, entre ellas el rol de la mujer en los pueblos prehispánicos, en este caso de las sociedades del noroeste argentino.

En una primera etapa fundacional las excavaciones sistemáticas en el noroeste argentino se realizaron a fines del siglo XIX, principios del XX, con el fin de conformar las colecciones arqueológicas de los dos principales museos surgidos aproximadamente en la misma época, el Etnográfico "J. B. Ambrosetti", dependiente de la Universidad de Buenos Aires, y el de Ciencias Naturales de la Universidad de La Plata. Estos primeros trabajos de campo y los que siguieron hasta aproximadamente los años 1960, se proponían recuperar en mayor medida restos arqueológicos de los contextos mortuorios, la cerámica principalmente; este interés por la funebria estaba enmarcado en los intentos de recuperar las piezas más completas del registro arqueológico y es justamente en los enterratorios donde se conservaban en ese estado. El enfoque era descriptivo / clasificatorio, se daba cuenta del tipo de entierro pero descontextualizando los hallazgos, en general no dejando registro escrito de las excavaciones realizadas y depositándolos luego en forma separada en distintos museos del país. Así, podemos mencionar las colecciones provenientes de La Paya (excavadas por Ambrosetti), de Quebrada de Humahuaca y Puna (excavadas por Debenedetti y Casanova), que se encuentran actualmente depositadas en el Museo Etnográfico J. B. Ambrosetti, dependiente de la facultad de Filosofía y Letras, UBA.

El tratamiento conjunto de los restos humanos y los objetos de acompañamiento en los contextos funerarios era variable, si bien en general para todos los investigadores recuperar los huesos dependía de su buena conservación. Aunque esta fuera buena, los investigadores no coincidían en un tratamiento riguroso ni en un protocolo, algunos (como es el caso de Debenedetti y Casanova) indicaban su disposición en el campo, y las edades aparentes pero recogían de forma parcial los restos óseos, de adultos en la mayoría de los casos, dejando in situ los de subadultos (Seldes 2007). Otros investigadores recolectaban solamente los cráneos, pero sin la exhaustiva descripción y relevamiento del acompañamiento como es el caso de Ambrosetti (Ambrosetti 1907). Finalmente una práctica habitual era la separación de las partes esqueletales colocando cráneos por un lado y huesos largos por el otro, sin conservar las conexiones anatómicas ni registro de las mismos, con lo cual se perdió la mayoría de las asociaciones contextuales (Seldes 2007).

Sin embargo hubo algunas excepciones como es el caso de los esqueletos provenientes del Pukará de Tilcara recuperados por Debenedetti, quien dirigió excavaciones de más de cien entierros entre 1905 y 1906. Los esqueletos que aún permanecen en conexión anatómica, fueron oportunamente analizados por Mendonça (et al. 1992). 
Las investigaciones, ya sean las realizadas bajo paradigmas descriptivos o los que le prosiguieron, realizados bajo los influjos de la escuela histórico - cultural (Baffi y Seldes 2011), sostuvieron un tratamiento separado de la evidencia, la arqueológica por un lado y la antropobiológica por otro. En estos trabajos la información bioantropológica a veces figuraba como apéndice con los resultados de los análisis pero sin discusión sobre los mismos. Un ejemplo lo constituye el estudio de los párvulos procedentes de los cementerios de San Blas de los Sauces en La Rioja (Boman 1927), el trabajo de Constanzó sobre cráneos de Pampa Grande (Constanzó 1941) o Paulotti sobre cráneos de Catamarca (Paulotti et al. 1949). En estos trabajos los resultados bioantropológicos no se discutían a la luz de los planteos y análisis arqueológicos, esto es, ninguna evidencia de un tipo servía para apoyar o contrarrestar evidencias de otro (Baffi y Berón 2000). Esto implicó en definitiva, una gran falta de información sobre los rituales mortuorios y otros aspectos sociales, como el tema de la especialización por sexo, por ejemplo.

En los trabajos arqueológicos, uno de los principales intereses estaba puesto en recuperar información sobre la cantidad de hombres y mujeres enterrados. Así, Ambrosetti (1907) en su trabajo sobre el sitio La Paya (Valle Calchaquí, provincia de Salta), al describir sus excavaciones, y ante la imposibilidad de reconocer el sexo biológico de los individuos, intenta determinarlo mediante los objetos depositados junto con los cuerpos, aplicando como criterio que el ajuar recuperado corresponde a los elementos utilizados por los individuos en vida. Consideraba a determinados objetos como típicamente femeninos y a otros de uso masculino, asignando la pertenencia de objetos cotidianos y/o suntuarios a uno u otro sexo, sin contemplar la asociación de todos los elementos del ajuar. A este investigador le resultó extremadamente difícil identificar objetos que le permitieran precisar el sexo de modo exacto en todos los casos, y creía que sólo era posible asegurar la presencia de alguna mujer en la tumba si ésta contenía uno o más torteros, a los que consideraba un útil propio de mujer. Entre los objetos que consideraba de uso masculino se cuentan las placas pectorales de cobre; en las tumbas las placas frecuentemente se encontraban asociadas a pucos negros. Así, al analizar el material cerámico planteó que los pucos negros han servido preferentemente

\begin{tabular}{|l|l|l|l|}
\hline OBJETOS & FEMENINO & MASCULINO & INDETERMINADO \\
\hline Calabazas & $\mathrm{X}$ & & \\
\hline Canastería & $\mathrm{X}$ & & \\
\hline Cinceles & $\mathrm{X}$ & & \\
\hline Escarificador & & & $\mathrm{X}$ \\
\hline Pintura roja & $\mathrm{X}$ & & \\
\hline Pinzas depilatorias & $\mathrm{X}$ & & \\
\hline Placas pectorales de cobre & & $\mathrm{X}$ & \\
\hline Pucos negros & & $\mathrm{X}$ & \\
\hline Puntas de proyectil & & $\mathrm{X}$ & \\
\hline Punzones grandes & & $\mathrm{X}$ & \\
\hline Resina de Yareta & $\mathrm{X}$ & & \\
\hline Tableta & & & $\mathrm{X}$ \\
\hline Tortero & $\mathrm{X}$ & & \\
\hline Vasos asimétricos & $\mathrm{X}$ & & \\
\hline
\end{tabular}

Tabla 1: Clasificación de objetos de acuerdo a Ambrosetti (1907) 


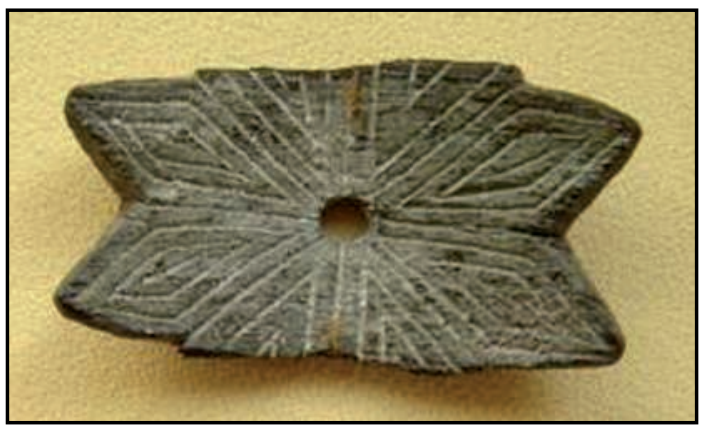

Figura 1. Objeto del mundo femenino: tortero

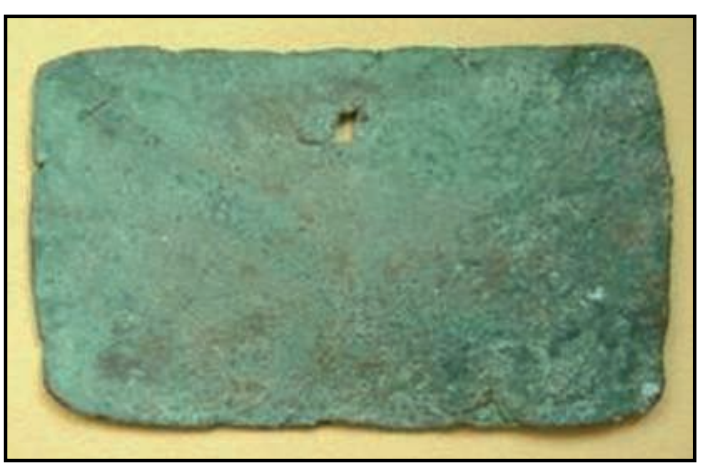

Figura 2. Objeto masculino: placa metálica a los hombres. También propuso que los punzones grandes serian de uso masculino, ya que habrían funcionado enmangados formando lanzas cortas. Por su parte a las mujeres las relacionaba con la presencia en la tumba de pinzas depilatorias, torteros, vasos asimétricos, pintura roja, cinceles, canastería, mates y cuentas de malaquita. En la tabla 1 se enumeran algunos objetos femeninos, masculinos e indeterminados de acuerdo a la clasificación realizada por Ambrosetti.

En síntesis, la información sobre el contenido de las tumbas de La Paya y su comparación con otros sitios para los que se cuenta con elementos para evaluar las diferenciaciones en los ajuares y su asociación al sexo de los inhumados, indica que las apreciaciones sobre la asignación sexual que hiciera Ambrosetti no resultan apropiadas para distinguir el/los sexos de los individuos enterrados en las tumbas. Esto podría resolverse con investigaciones específicas de los cuerpos en relación con su acompañamiento, ya sea en este sitio (si fuera posible rearmar los contextos) o en otros con áreas de entierro estrechamente relacionadas (Baffi y Baldini 2010).

En la misma línea de pensamiento de Ambrosetti, un ejemplo reciente lo constituye el trabajo de Palma (1997/98) en su análisis del comportamiento mortuorio en el sitio La Huerta (Quebrada de Humahuaca, Jujuy). A partir de las libretas de campo de Debenedetti, infiere diferencias de rango por el acompañamiento mortuorio y parte de la concepción que ese acompañamiento perteneció a la persona allí enterrada. A su vez, infiere el sexo de las personas enterradas en las tumbas a partir del ajuar recuperado; de esta manera se trataría de mujeres si las personas se encuentran asociadas a topos y útiles de hilado como husos y torteros y hombres los que están acompañados de objetos asociados a prácticas bélicas (Palma 1997/98).

Algunas dificultades se registran en esta dinámica asociativa; en primer lugar la asignación a determinado objeto como de uso exclusivo de un sexo, es que opera como un sistema de identificación mecánico, esto es, siempre que se encuentra tal objeto se trata de tal sexo. En palabras de Alberti: "El resultado de concebir macho y hembra como interiores y centrales de la identidad es que la cultura material, los restos de esqueletos y las representaciones figurativas de los cuerpos se entienden simultáneamente como las expresiones de género y como las evidencias para ese género" (Alberti 2001: 69).

Por otra parte este tipo de razonamientos no toman en cuenta las complejidades de los rituales funerarios; en este sentido los restos que acompañan al difunto podrían no haber 
pertenecido a él sino ser parte de una serie de actos de depositación de ofrendas que responden a diversas relaciones y posiciones sociales y no necesariamente una asociación directa entre individuo y ajuar (Carr 1995).

\section{La mujer en el registro arqueológico, nuevas perspectivas}

Con la incorporación de nuevas perspectivas teóricas a partir del afianzamiento de la escuela procesual en los años setenta, la disciplina se fue flexibilizando y dando lugar al surgimiento de diversas corrientes teóricas que persisten hasta el día de hoy el trabajo arqueológico (Alberti 2001, Politis 2001, 2006) y bioarqueológico.

Sin embargo, realizando una lectura general de la producción antropobiológica del Noroeste argentino durante los últimos diez años, considerando el auge de las teorías denominadas postprocesuales, en especial la teoría de la práctica y la agencia y los estudios de arqueología de género, no se registra una preocupación por temáticas como el mundo femenino o el rol de la mujer en las sociedades prehispánicas.

Recapitulando entonces, el recorrido por la historia de la disciplina, las mujeres han sido prácticamente invisibles en la reconstrucción del pasado arqueológico del noroeste argentino. Relevando la producción en la antropología biológica o en ámbitos cercanos a ella como el estudio de las prácticas mortuorias realizados por arqueólogos, solo se han registrado algunos trabajos en los últimos años. Un ejemplo es el análisis de un esqueleto femenino realizado por Scattolin a efectos de realizar inferencias mas generales sobre las sociedades que habitaron el valle de Yocavil (Scattolin et al. 2005), y aquel donde también Scattolin trabaja, a partir de las hidroánforas en tanto recipientes antropomorfos representando mujeres, para señalar la invisibilización de las imágenes femeninas poco exploradas por la arqueología argentina frente al énfasis colocado por los investigadores en las figuras de hombres en tanto guerreros (Scattolin 2006, Williams y Alberti 2006).

Un punto importante a tener en consideración es que en general los trabajos antropobiológicos se plantean la discriminación de la estructura poblacional en cuanto a sexo y edad y realizan inferencias sobre la organización social de los pueblos prehispánicos incluyendo la división sexual del trabajo. Sin embargo, como se ha demostrado, prácticamente no se registran trabajos con claras intenciones de rescatar el mundo femenino en la prehistoria.

En cuanto a los estudios específicos de arqueología de género, existen pocos trabajos en nuestro país, y éstos se refieren principal y casi exclusivamente a cuestiones de la práctica profesional; en ellos se señalan diferencias de género en cuanto al acceso al trabajo de campo y su organización interna, o bien se resaltan las desigualdades en el acceso a posiciones alcanzadas en organismos de investigación o en las universidades (Belleli et al. 1993, Gero 1996, Navarrete 2010, Williams y Alberti 2006).

\section{Lo femenino en el mundo andino}

Antes de continuar con la reflexión acerca de lo que se ha escrito hasta ahora respecto al mundo femenino a partir de las prácticas funerarias, se describirá el vínculo entre el mundo de los vivos y los muertos establecido a partir de los trabajos arqueológicos y antropobiológicos a efectos de contextualizar el comportamiento mortuorio en el NOA. 
Una de las principales características de la mayoría de los entierros en quebrada de Humahuaca y valle Calchaquí (así como en el resto del NOA), es que dan cuenta de una cercana convivencia de espacios domésticos y funerarios; estos últimos se ubican principalmente en los patios de las viviendas. Numerosos sitios arqueológicos dan cuenta de esto: Los Amarillos, Juella, Huichairas en quebrada de Humahuaca (Lafón 1958/59, 1967, Nielsen 2001, Seldes 2007). Por su parte en el caso del valle Calchaquí, durante el Periodo Tardío, se encuentran espacios mortuorios exclusivos, en contacto estrecho con unidades habitacionales, como La Paya, Molinos I, entre otros (Baldini y Baffi 2007, 2008). De esta manera los pueblos prehispánicos que habitaron el Noroeste Argentino mantuvieron una convivencia cercana con espacios funerarios, los cuales, en general, no estaban segregados espacialmente de las viviendas.

Si bien esto se da de manera generalizada, se han registrado espacios específicos para entierros (necrópolis) como los registrados en los Pukará de Tilcara y Volcán, ambos sitios de la quebrada de Humahuaca del período Tardío e Inka (Debenedetti 1930, Gatto 1946) y el cementerio de Rincón Chico en el valle de Yocavil (Tarrago et al. 2005). Este tipo de prácticas constituyen casos excepcionales que han sido asociados al afianzamiento de sociedades de tipo corporativas y la necesidad de garantizar un territorio por medio del vínculo establecido con los antepasados (Seldes 2010). Por su parte también en el valle Calchaquí se han registrado espacios funerarios segregados, en este caso para el período Formativo en

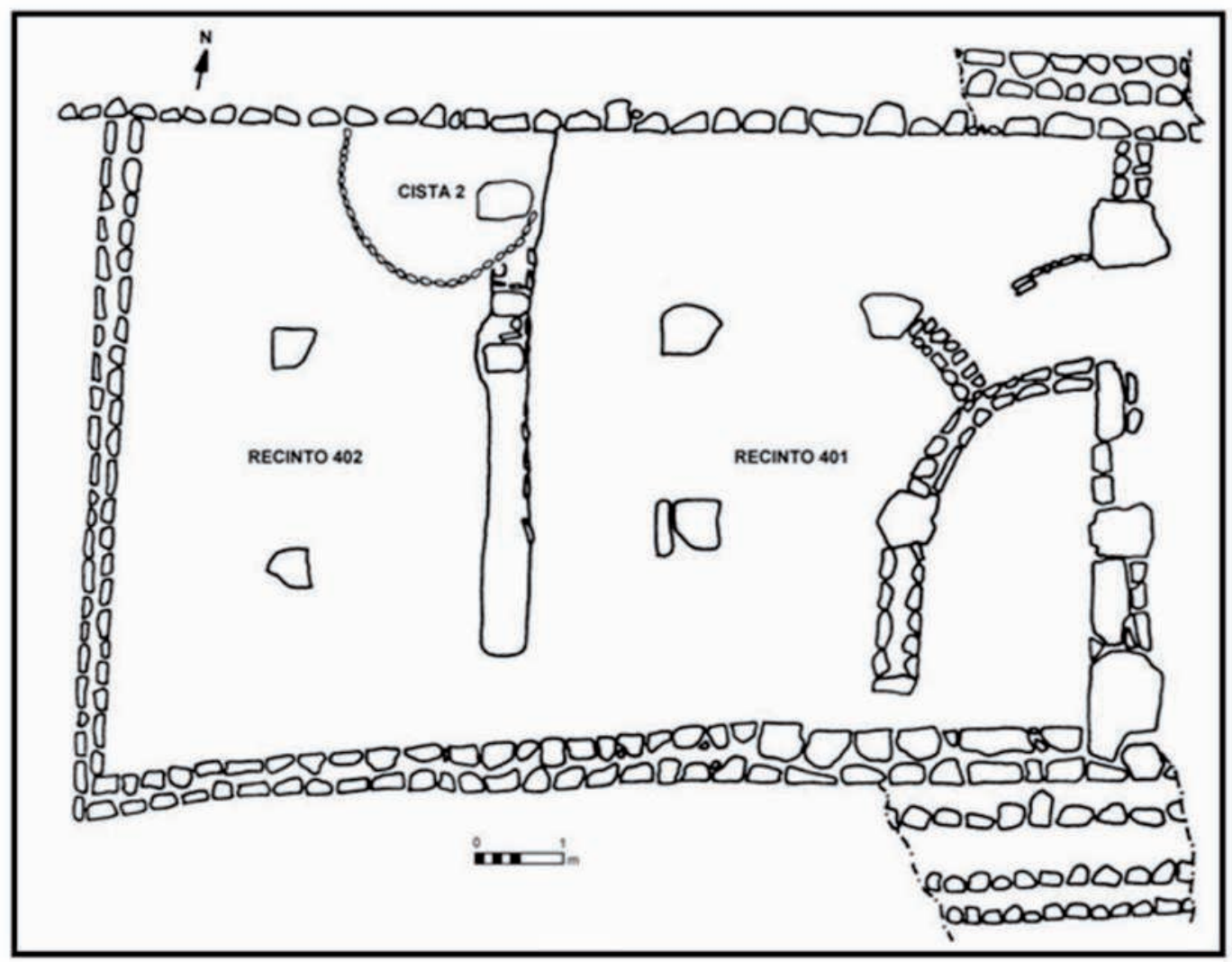

Figura 3. Cista en espacio doméstico, sitio Los Amarillos, unidad 400 (Angiorama 2006) 
sitios como Salvatierra y Cancha de Paleta, fenómenos estos últimos que aún requieren de mayores análisis desde una perspectiva regional (Baldini 2007, Seldes y Zigarán 2010).

La cercanía entre los mundos de los "vivos" y los "muertos", cristalizada en la escasa presencia de cementerios en el NOA, se registra en diferentes dimensiones socio-políticas; por un lado, se ha hecho referencia a la importancia de los difuntos en la vida cotidiana a través de la convivencia de espacios domésticos y funerarios. Por otro lado, algunos antepasados habrían tenido gran preponderancia ya que se convertirían en "ancestros".

Nielsen entiende el culto a los ancestros como "un conjunto de prácticas religiosas que permiten la intervención de los muertos en los asuntos de los vivos" (Nielsen y Boschi 2007: 52). Este tipo de culto que se registra en numerosas sociedades es considerado una forma de memoria colectiva (Nielsen 2008). Los ancestros eran considerados los fundadores de los ayllus, propietarios originales de las tierras y fuente ultima de toda autoridad, eran los encargados de mantener el bienestar de la comunidad así como garantizar las cosechas y el ganado (Nielsen y Boschi 2007). Los ancestros eran componentes fundamentales de la reproducción de la sociedad (Hastorf 2008) como generadores y reforzadores de identidades (Nielsen 2008).

En diferentes escalas y dimensiones, es posible señalar que el mundo de los muertos en general constituía un punto sumamente sustancial para la organización social de los pueblos, tanto por la importancia adquirida por aquellos convertidos en "ancestros" y venerados como fundadores de ayllus, como en la convivencia cercana con los antepasados directos (Nielsen y Boschi 2007).

Dada la naturaleza de las fuentes etnohistóricas y las dificultades para diferenciar aspectos masculinos y femeninos en el registro arqueológico (aspecto que se retomará mas adelante), no es de extrañar que la mujer no aparezca constituida como actor social y político (Escoriza Mateu 2002) en los relatos sobre ese mundo que se ha denominado "de los muertos". Pero esto no es exclusivo de los contextos funerarios ya que, de acuerdo al recorrido por la arqueología que se ha realizado, la mujer prácticamente tampoco aparece en las descripciones sobre el mundo de "los vivos".

\section{La bioarqueología y la mujer}

Una vez recorrido el escenario de las producciones académicas sobre prácticas mortuorias, se focalizará en lo que se ha escrito respecto a la mujer desde los análisis bioarqueológicos propiamente dichos, siempre en referencia al área seleccionada para este trabajo, el NOA.

La violencia y la mujer

A continuación se realiza una revisión de algunos trabajos donde se intenta rescatar al mundo femenino en los pueblos prehispánicos que habitaron el noroeste argentino.

Un ejemplo clásico sobre la fuerte ponderación del universo masculino frente al femenino es el análisis de cráneos trofeo que realiza Vignati (1930). De acuerdo a este investigador los cráneos trofeo correspondían a individuos masculinos en su totalidad. En sus propias palabras: "... todos esos cráneos corresponden a individuos del sexo masculino, con exclusión absoluta del otro sexo, lo que se explica dado el carácter de guerreros que les he atribuido." (Vignati 1930: 60). 
Sin embargo trabajos posteriores contradicen fuertemente este tipo de interpretaciones. Bordach relevó los cráneos analizados por Vignati y observó la presencia de cráneos trofeos correspondientes a individuos femeninos (Palma 1997/98). Por otra parte resultados similares a los de Bordach se obtuvieron al analizar cráneos de la Quebrada de Humahuaca depositados en el Museo Etnográfico "J. B. Ambrosetti", registrando individuos femeninos entre ellos (Gheggi y Seldes en prensa)

Por consiguiente, los recientes análisis estarían evidenciando que las mujeres tuvieron algún tipo de intervención y/o participación en situaciones de violencia social, al menos durante los Períodos de Desarrollos Regionales (900 - 1250 dC.) e Inka (1250 - 1430 dC.).

También ejemplos de otras regiones del NOA contradicen las interpretaciones realizadas por Vignati. En el caso de Las Pirguas (Pampa Grande, Salta), adscripto a la cultura de La Candelaria (con fechados radiocarbónicos que la ubica alrededor del $500 \mathrm{dC}$.), se registraron evidencias de violencia interpersonal siendo que no se encontró una clara diferenciación por sexo; se observaron golpes en el cráneo en al menos 10 individuos sobre un total de 23 varones y 11 entre 27 mujeres. Las señales de agresión parecen haber sido producidas por armas redondeadas y pesadas, que produjeron golpes circulares en la parte posterior del parietal izquierdo. Los niños presentan también las mismas lesiones lo cual da cuenta de la no discriminación por grupos de edad o sexo (Baffi et al. 1996).

A partir de estas reflexiones sobre la participación efectiva de la mujer en situaciones de violencia social, interesa aquí resaltar que en ese universo denominado Andes Meridionales de los cuales el NOA forma parte, no se registra un patrón único sino que la misma tuvo características particulares en la heterogeneidad que representa la región.

Y en ese heterogéneo mundo prehispánico, uno de los puntos fundamentales a evaluar es si existían diferencias en el estilo de vida entre hombres y mujeres, uno de los pocos tópicos en los cuales la antropología biológica sí ha focalizado su análisis. En este sentido, el principal problema que se registra para su abordaje radica en las dificultades que se presentan a la hora de recuperar muestras lo suficientemente amplias como para poder realizar comparaciones entre ambos sexos. Las colecciones del Museo Etnográfico, salvo excepciones, no cuentan con esqueletos completos con lo cual solo es posible evaluar los cráneos por un lado y el esqueleto postcraneal por el otro, generando una gran pérdida de información.

Sumado a esto los individuos recuperados en excavaciones en los últimos años, resultan escasos o su estado de conservación dificulta la determinación de sexo (Seldes 2007).

Ahora bien, con los recaudos necesarios frente a las dificultades para determinar sexo en una cantidad suficiente de individuos, se propone un repaso de algunos de los análisis realizados a efectos de evaluar cuánto ha podido avanzarse en el análisis de patrones de comportamiento y estilo de vida por sexo en el NOA.

Quebrada

Ubicada en el sector central de la provincia de Jujuy, la quebrada de Humahuaca presenta evidencias de ocupación desde tiempos arcaicos (Nielsen 2001). A partir de esto y hacia el período Formativo Tardío (500 - 900 dC.) se dieron una serie de cambios que transformaron a las pequeñas formaciones sociales sin desigualdades estructurales, asentadas en poblados dispersos o semiconglomerados, en sociedades internamente diferenciadas a partir del Período de Desarrollos Regionales (900 - 1250 dC.), concentradas a lo largo de la quebrada 
troncal en los denominados Pukaras, con una gran intensificación de la producción, la aparición de conflictos interregionales y una fuerte organización a través de grupos corporativos (Nielsen 2001, 2006a).

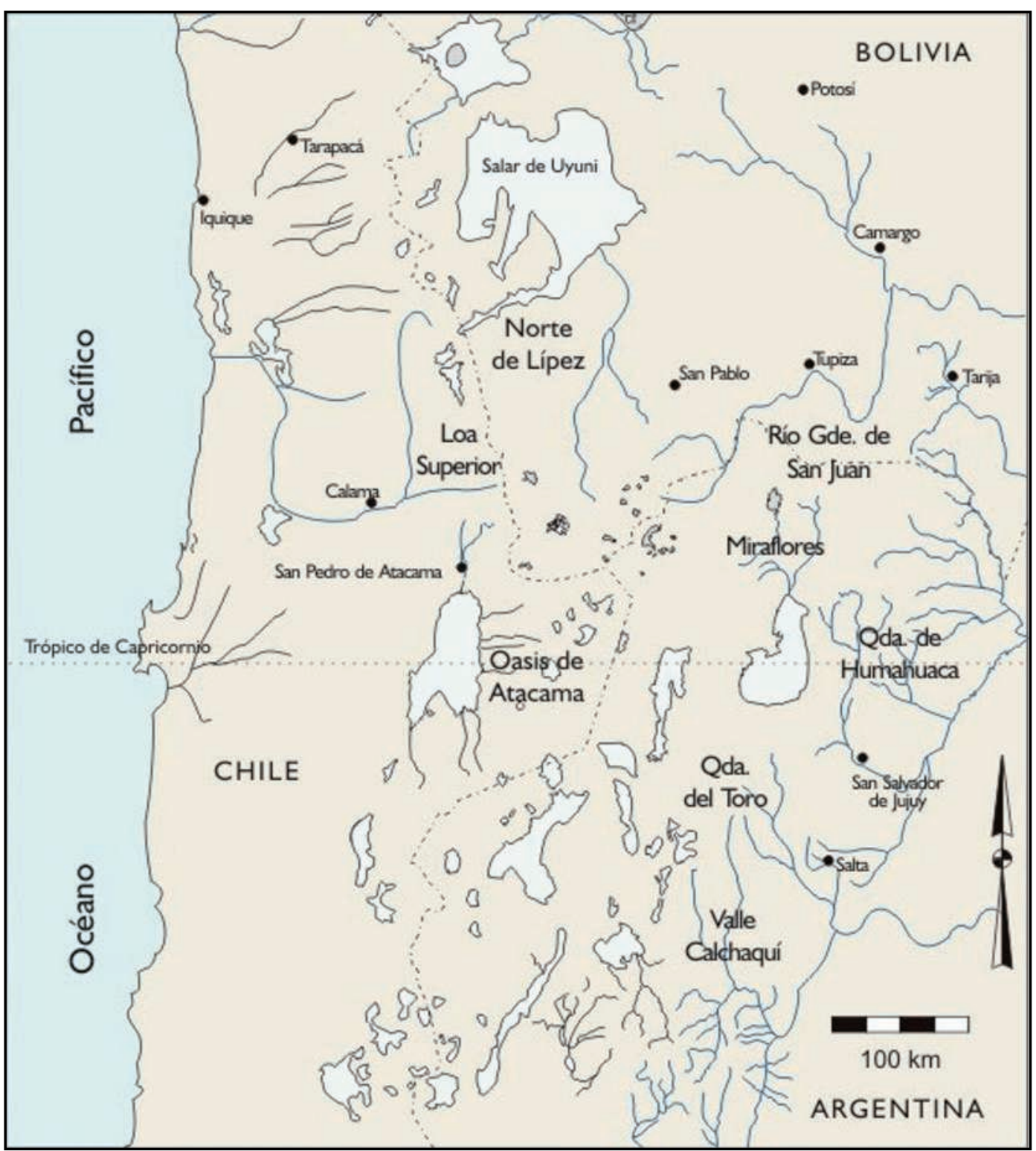

Figura 4. Ubicación de la quebrada de Humahuaca y valle Calchaquí (Nielsen 2006b)

La poca cantidad de individuos recuperados de forma completa impide la realización de inferencias sobre determinadas cuestiones entre las cuales se cuenta la diferencia en el estilo de vida entre hombres y mujeres. Sin embargo se han realizado algunos análisis bioarqueológicos correspondientes a diferentes períodos de ocupación y que constituyen un punto de partida que permite ir acumulando información a efectos de evaluar las posibles diferencias sociales. 
Así, en los períodos Formativo Tardío (500 - 900 dC.) y Desarrollos Regionales Temprano (900 - 1250 dC.), en sitios como Flores, Til 20 y Muyuna, sobre una muestra de 11 individuos femeninos y 6 masculinos, no se registraron diferencias en el estilo de vida entre ambos sexos relevando indicadores bioarqueológicos que dieran cuenta del estrés nutricional - metabólico, estrés funcional y traumas (Bordach et al. 1999, Mendonça et al. 1991, 2002, Seldes 2007).

Los cambios dados a partir del nuevo reordenamiento social principalmente en el Período de Desarrollos Regionales Tardío (1250 - 1430 dC.) fueron analizados por Seldes en los sitios Los Amarillos y Hornillos concluyendo que, si bien se trata de una muestra poco numerosa (14 hombres y 10 mujeres), no se registraron diferencias significativas entre hombres y mujeres en lo referido al acceso a recursos, estrés nutricional-metabólico, estrés funcional y traumas (Seldes 2007).

Sin embargo, Mendonça en el sitio Til 1 (Pukará de Tilcara) sobre un total de 12 individuos masculinos y 16 femeninos, registra algunas asimetrías en los patrones de dieta, estrés funcional y traumas entre hombres y mujeres e infiere actividades diferenciales para cada uno de los sexos (Mendonça et al. 1992).

Respecto al período Inka (1430 - 1536 dC.), el trabajo de Gheggi sobre el sitio Esquina de Huajra sugiere que, sobre una base de 5 individuos masculinos y 7 femeninos, los hombres desarrollaron actividades cotidianas que involucraban un uso mas generalizado del cuerpo que las mujeres, estas últimas habiendo realizado un uso intensivo de los miembros superiores (Gheggi 2005).

Son evidentes las dificultades que el reducido tamaño muestral imponen para realizar inferencias a nivel poblacional sobre diferencias en el estilo de vida entre hombres y mujeres. Sin embargo se señala el potencial de este tipo de trabajos y el constante incremento de casos, lo cual redundará en la posibilidad de trabajar con este tipo de problemáticas con mayor nivel de profundidad.

\section{Valle Calchaquí}

En este caso se realizó un trabajo de gran amplitud pero limitado al análisis de cráneos; se analizaron 382 individuos procedentes de siete sitios arqueológicos (La Paya, La Poma, Luracatao, Cachi, Fuerte Alto, Payogasta y Tacuil). Los mismos, de acuerdo con trabajos anteriores, en los cuales se los comparó por su variabilidad intrínseca y sus relaciones biológicas con poblaciones mas o menos contemporáneas procedentes de distintas regiones del Noroeste Argentino, constituyeron una población biológicamente diferenciada, durante el período de Desarrollos Regionales (1000-1450 dC. ${ }^{1}$ ) (Baffi 1992, 2011).

En esta población se analizó la variabilidad intragrupal, teniendo en cuenta los efectos del sexo, la edad y la deformación artificial sobre las variables métricas del cráneo relevadas según los criterios de la Convención Internacional de Mónaco de 1908 y sus posteriores modificaciones (Rodríguez Cuenca 2004), aplicando técnicas estadísticas.

Por otra parte se consideraron aspectos biosociales de la población, como la violencia intra-intergrupal a partir de lo evidenciado por marcas de golpes y otras señales de agresión. Se analizó la variabilidad entre los distintos sexos, mediante el coeficiente de variación, y esas diferencias se interpretaron según distintas posibilidades de las pautas de residencia postmarital. 
Con respecto a la variabilidad métrica, las mujeres presentaron menor variabilidad que los individuos varones (Baffi 1992, 2011). Esto puede relacionarse con lo propuesto por diferentes autores (Koninsberg 1988, Lane y Sublett 1972) que intentan, a partir de la variabilidad entre sexos, aproximarse a pautas de residencia postmarital. De acuerdo a esto, nuestra población podría incluirse en un modelo exogámico, con residencia posterior virilocal. La libre circulación de las mujeres se vincularía con un factor microevolutivo como la deriva, que favorecería el proceso dispersivo de los varones, causante de su mayor variabilidad (Baffi 1992, 2011).

Tomando otros ejemplos, para el período Formativo (500 aC. - 1000 dC.), se cuenta con los esqueletos provenientes del sitio Salvatierra (Salcac91) excavado por Pio Díaz en la década del 70 y analizados por Seldes y Zigarán. La muestra se encuentra en mal estado de conservación por lo cual no han podido evaluarse sus frecuencias de aparición entre hombres y mujeres ya que la cantidad de individuos analizados resulta muy pequeña, 5 mujeres y 4 hombres (Seldes y Zigarán 2010). Esto demuestra una vez más las dificultades de contar con muestras amplias y completas para realizar comparaciones.

\section{Reflexiones finales}

Este trabajo se propuso realizar aportes a una línea de investigación poco desarrollada en nuestro país; en este caso se ha elegido comenzar realizando una revisión de los trabajos sobre la mujer en el registro bioarqueológico para reflexionar sobre el vacío que hay en este sentido.

La historia de la disciplina nos ha mostrado una lenta incorporación de perspectivas teóricas denominadas postprocesuales como son las teorías de la práctica, de la agencia y las perspectivas de género, a pesar de la importancia que las mismas tienen en otras áreas afines como la arqueología.

Esto podría estar reflejando resistencias por parte de la antropología biológica a la incorporación de nuevos paradigmas teóricos (Baffi y Seldes 2011, Geller 2008) que incluyan entre otros aspectos, aquellos vinculados a las lógicas culturales y prácticas sociales de los pueblos prehispánicos y que permitan "establecer necesarios vínculos entre los datos bioarqueológicos y la naturaleza sociocultural del grupo observado (Seldes 2009).

En muchas ocasiones a lo largo de la historia de la disciplina, los estudios bioantropológicos y los arqueológicos se mantuvieron como compartimentos estancos sin conexión entre sí; esta desarticulación llevó a dificultades en la identificación de diferencias en los rituales mortuorios entre hombres y mujeres, los bioantropólogos estudiaban los restos óseos independientemente del contexto mortuorio mientras que los arqueólogos describían y analizaban los restos arqueológicos sin combinarlos con los resultados obtenidos por los bioarqueólogos.

Entre los temas abordados y los ejemplos brevemente descriptos en este trabajo, puede inferirse para el caso de las situaciones de violencia social, que no habría habido una clara asignación de roles regidas por cuestiones de sexo; sin embargo, las investigaciones arqueológicas en general aceptaron de manera acrítica que las mujeres y los niños no habrían guerreado ni participado de situaciones de conflicto social, siendo los hombres los encargados de resolver en ocasiones por medio de la violencia los problemas intergrupales. 
Llegados a este punto es interesante recuperar la bucólica imagen de las sociedades prehispánicas con una marcada tipificación en la división de roles: las mujeres recolectan y los hombres cazan en los grupos cazadores recolectores (Wylie 1997), las mujeres cultivan y confeccionan piezas cerámicas, mientras los hombres guerrean en las sociedades agropastoriles (Scattolin 2006).

Esto podría estar respondiendo a que las interpretaciones de la evidencia arqueológica estuvieron durante muchos años imbuidas de los propios modelos culturales y estereotipos sobre los roles de la mujer y del hombre en la propia sociedad (Gilchrist 1999), así como su participación en eventos de conflicto interpersonal que atraviesan y permean los análisis. Probablemente es este imaginario social el que ha influido en las asociaciones objetos-sexo referidas anteriormente. Al realizar esta interpretación se utilizaban objetos paradigmáticos femeninos, o extraídos de la propia realidad cotidiana (Baffi y Baldini 2010).

Sin embargo, hoy en día se postula que estas referencias cuasi universales a los roles asociados a cada sexo, parten de categorías sociales (Balmer y Bowdler 2006) que subsumen la heterogeneidad que se registra en las diferentes sociedades a lo largo del tiempo y del espacio. No hay nada biológico en estas formas de organización social sino que se trata de construcciones donde el sexo en tanto categoría biológica, esto es, ciertas características universales del cuerpo, aparece naturalizando (Gilchrist 1999) los que son categorías elaboradas culturalmente.

La propuesta de este trabajo es señalar la necesidad de acercar los trabajos bioarqueológicos con las problemáticas arqueológicas referidas a las prácticas socioculturales de los pueblos y los cambios que se fueron dando en su desarrollo. Sólo a partir de esto se considera posible realizar una aproximación al rol de la mujer en las sociedades del NOA.

Aquí conviene realizar algunas aclaraciones respecto a la terminología utilizada. La referencia a "las sociedades del NOA" no se realiza en el sentido de considerar que constituyan una entidad única y homogénea. No se pretende establecer relaciones estructurales entre ambos sexos y generalizables al NOA, lo cual sería caer en la misma esencialización que se está cuestionando, sino que se trata de profundizar el análisis de las lógicas culturales de cada uno de los pueblos reconociendo que se trata de un universo altamente heterogéneo. Por consiguiente no es cuestión de extrapolar características de un grupo obtenidas a partir de datos arqueológicos o etnohistóricos a otros, sino ver la especificidad de cada uno, tal como se ha tratado de mostrar en este trabajo.

En este sentido, Escoriza Mateu realiza una interesante propuesta referida al análisis de las representaciones figurativas de la mujer en la zona del levante español: "Only by analysing the material conditions of the communities that produce and/ or give meaning to the figurative representations can we explain them. Thus, it becomes necessary to establish transitive relations between the conditions of the production of social life (social practices) and their material, archaeological expression" (Escoriza Mateu 2002: 87).

En este camino, en especial en referencia a la cuestión de las ofrendas y/o el acompañamiento funerario, luego de haber discutido la asignación del sexo del difunto por la cualidad de determinado acompañamiento y los estereotipos que la ciencia ha seguido en este derrotero (Gilchrist 1999), se propone seguir otro rumbo. 
Se considera pertinente trabajar sobre la información que provee el registro bioarqueológico referido al sexo de los individuos, asociarlos con los objetos registrados como acompañamiento funerario y con esto profundizar en los estudios específicos de cada uno de los pueblos que habitaron el NOA en tiempos prehispánicos, en especial en lo referido a pautas de organización social, de prácticas cotidianas y de los rituales funerarios, intentando "visibilizar" a la mujer, su estar en el mundo, analizar sus prácticas cotidianas desde una perspectiva que des-universalice la conocida división sexual del trabajo (Navarrete 2010).

No se pretende sostener una posición de relativismo extremo sino proponer que en el camino abierto por las nuevas corrientes teóricas, como las teorías de la agencia y la práctica (Seldes 2007), la antropología biológica en Argentina puede tomar nota, y de hecho lo viene haciendo en los últimos años, de la necesidad de no esencializar las identidades, incluyendo las femeninas o masculinas, considerando que las mismas son relacionales y que se comprenden en su contexto de producción y reproducción mas que a partir de leyes generales que permitan interpretar los contextos mortuorios sin tener en cuenta su historia particular (Fowler 2004). En palabras de Alberti:

"Otra implicación de no tener que buscar las categorías invariables de macho y hembra en el registro arqueológico es que los estudios funerarios dejan de ser una búsqueda desesperada de la verdadera identidad sexual de los cadáveres enterrados y pueden explorar otras posibilidades de cómo la gente en el pasado conformaba sus identidades y relaciones a través de sus prácticas fúnebres" (Alberti 2001: 69).

\section{Agradecimientos}

Agradecemos a los evaluadores anónimos y al comité editorial por sus sugerencias y comentarios. Sus aportes nos permitieron profundizar en algunos puntos importantes de este trabajo.

\section{Notas}

1. Las diferencias interregionales para cada período corresponden a las evidencias y las consiguientes variaciones en las periodificaciones propuestas por distintos investigadores. Siendo que no es el objetivo del trabajo realizar comparaciones interregionales, prefirió respetarse la especificidad planteada para cada región.

\section{Bibliografía citada}

Alberti, B.

2001 De género a cuerpo: una reconceptualización y sus implicaciones para la interpretación arqueológica. Intersecciones en Antropología 2: 61 - 72.

Ambrosetti, J.

1907 Exploraciones arqueológicas en la ciudad prehistórica de La Paya (Valle Calchaquí) Publicación Sección Antropología FFyL 3. Universidad de Buenos Aires.

Angiorama, C.

2006 ¿Mineros quebradeños o altiplánicos? La circulación de metales y minerales en el extremo noroccidental de Argentina (1280-1535 AD). Intersecciones en Antropología 7: 147 - 161. 
Baffi, E. I.

1992 Caracterización biológica de la población prehispánica tardía del sector septentrional del valle Calchaquí (Pcia. de Salta). Tesis de doctorado. Facultad de Filosofía y Letras, Universidad de Buenos Aires.

2009 De la crania étnica a la paradoja osteológica. Apuntes a los avances de la Bioarqueología en Argentina. IX Jornadas de Antropología Biológica. Puerto Madryn.

2011 La población prehispánica tardía del sector septentrional del valle Calchaquí (provincia de Salta, Republica Argentina). ANTHROPOS II Congreso Iberoamericano de Antropología. Memorias: 1138-1154 (CD. ISBN 978-959-7091-77-6). La Habana, Cuba.

Baffi, E. I. y L. Baldini

2010 Otra dimensión de las tumbas de La Paya: sexo y objetos en relación. En Arqueología Argentina en el Bicentenario de la Revolución de Mayo. (ed. por R. Barcena. y H. Chiavazza), tomo II, pp. 653-658. Facultad Filosofía y Letras, UNCuyo, Instituto de Ciencias Sociales, Humanas y Ambientales, CONICET.

Baffi, E. I. y M. Berón

2000 Propuesta para la integración del conocimiento de los sitios arqueológicos con restos humanos. El sitio Chenque I, provincia de La Pampa. Relaciones de la Sociedad Argentina de Antropología XXV: 145-158.

Baffi, E I. y V. Seldes

2011 Aportes osteobiográficos al conocimiento de las poblaciones prehispánicas del Noroeste Argentino. ANTHROPOS II Congreso Iberoamericano de Antropología. Memorias: 2821-2823. La Habana, Cuba.

Baffi, E., M. F. Torres y J. A. Cocilovo

1996 La población prehispánica de Las Pirguas (Salta). Un enfoque integral. Revista Argentina de Antropología Biológica 1 (1): 204-218.

Baldini, L.

2007 Cancha de Paleta: un cementerio del período formativo en Cachi (Valle Calchaquí, Salta). Cuadernos de la facultad de Humanidades y Ciencias Sociales 32: 13 - 33. Universidad Nacional de Jujuy, Argentina.

Baldini, L y E. I. Baffi

2007 Muertos y vivos. Comportamiento mortuorio de sociedades del valle Calchaquí (provincia de Salta, Argentina) durante el Período de Desarrollos Regionales. ANTHROPOS I Congreso Iberoamericano de Antropología. Memorias: 3260-3270 (CD. ISBN 959-282-043-0). La Habana, Cuba.

2008 Modos funerarios y alimentación. El caso de Molinos I, valle calchaquí, Salta, Argentina. ALER XII Congreso Latinoamericano de Religión y Etnicidad, Bogotá, Colombia: 44-56.

Balmer, J. y S. Bowdler

2006 Spear and dikking stick. The origin of gender and its implications for the colonization of new continents. Journal of Social Archaeology 6 (3): 379 - 401.

Belelli, C., V. Scheinsohn y M. Berón

1993 Una arqueología de distinto género. Publicar 2 (3): 47-61. 
Boman, E

1927 Estudios Arqueológicos Riojanos. Anales del Museo Nacional de Historia Natural Bernardino Rivadavia 35: 7-68.

Bordach, M. A., L. Dalerba L. y O. Mendonça

1999 Vida y Muerte en Quebrada de Humahuaca. Universidad Nacional de Río Cuarto. Córdoba.

Carnese, F., J. Cocilovo J. y A. Goicochea

1997 Argentina. History of Physical Anthropology 1: 101-107. Ed. Garland publishing. New York y London.

Carr, C.

1995 Mortuary practices: their social, philosophical - religious, circumstancial, and physical determinants. Journal of Archaeological Method and Theory 2: 105 - 200.

Constanzó, M.

1941 Restos humanos de Pampa Grande Anales Instituto Etnografía Americana 2: 239-264. Mendoza.

Debenedetti, S.

1930 Las ruinas del Pucará, Tilcara, Quebrada de Humahuaca (Provincia de Jujuy). Archivos del Museo Etnográfico II: 7 - 142. Facultad de Filosofía y Letras, Universidad de Buenos Aires.

Escoriza Mateu, T.

2002 Representations of women in Spanish Levantine rock art. Journal of Social Archaeology 2 (1): $81-108$.

Fowler, Ch.

2004 Archaeology of Personhood. An Anthropological Approach. Ed. Routledge, London and New York.

Gatto, A.

1946 Exploraciones arqueológicas en el Pucará de Volcán. Revista del Museo de La Plata 4: 5 - 91. La Plata.

Geller, P.

2008 Conceiving sex: Fomenting a feminist bioarchaeology. Journal of Social Archaeology 8 (1): $113-138$.

Gero, J.

1996 Archaeological practice and gendered encounters with field data. Gender and Archaeology (ed. por R. Wright), pp. 251-279. Filadelfia, University of Pennsylvania Press.

Gheggi, M. S.

2005 Análisis bioarqueológico y contextual en enterratorios arqueológicos. Un caso de estudio en Esquina de Huajra (Quebrada de Humahuaca- Jujuy, Argentina). Tesis de Licenciatura, FFyL, UBA. Buenos Aires. 
Gheggi, M.S. y V. Seldes

2012 Evidencias bioarqueológicas de conflicto ca. 1000-1432 A.D. en el Valle Calchaquí y la Quebrada de Humahuaca. Intersecciones en Antropología, en prensa

Gilchrist, R.

1999 Gender and Archaeology. Contesting the Past. Routledge. London and New York.

Hastorf, C.

2008 Construyendo comunidad, ceremonialismo y memoria social en el Formativo Medio de Chiripa, Bolivia. Arqueología de las tierras altas, valles interandinos y tierras bajas de Bolivia, memorias del I Congreso de Arqueología de Bolivia (ed. por C. Rivera Casanovas), pp. 3 - 12. La Paz, Bolivia.

Konigsberg, L.W.

1988 Migration models of prehistoric postmarital residence. American Journal of Physical Anthropology 77: 471-482.

Lafon, C. R.

1958/59 Ensayo sobre cronología e integración de la cultura Humahuaca. Runa IX: 217 230.

1967 Un estudio sobre la funebria Humahuaca. Runa X: 195 - 255

Lane, R. y A. Sublett

1972 Osteology of social organization: residence pattern. American Antiquity 37: 186 - 200.

Mendonça O., M. A. Bordach, M. Grosso y L. Dalerba

2002 Ambiente, comunidad y comportamiento biosocial en el Formativo de Tilcara, Quebrada de Humahuaca, Jujuy. Pacarina 2 (2): 135 - 147. Universidad Nacional de Jujuy. San Salvador de Jujuy.

Mendonça O., M. A. Bordach, M. Ruiz y B. Cremonte

1991 Nuevas evidencias del período agroalfarero temprano en Quebrada de Humahuaca. Los hallazgos del sitio Til 20 (Tilcara, Jujuy). Comechingonia 7: 31 - 45. Córdoba.

Mendonça, O., M. A. Bordach y S. Valdano

1992 Reconstrucción del comportamiento biosocial en el Pukará de Tilcara (Jujuy). Una propuesta heurística. Cuadernos 3: 144 - 151. Facultad de Humanidades y Ciencias Sociales, Universidad Nacional de Jujuy. San Salvador de Jujuy.

Navarrete, R.

2010 Excavando mujeres en y desde el sur: aproximaciones a la arqueología feminista en Latinoamérica. Revista venezolana de estudios de la mujer 15 (34): 75 - 104.

Nielsen, A. E.

2001 Evolución social en Quebrada de Humahuaca (AD 700-1536). En Historia Argentina prehispánica (ed por A. Nielsen y E. Berberián), tomo I, pp. 171-264. Editorial Brujas, Córdoba.

2006a Pobres Jefes: aspectos corporativos en las formaciones políticas preincaicas de los andes circumpuneños. Contra la tiranía tipológica en arqueología: una visión desde Sudamérica (ed. por C. Langebaek y C. Gnecco), pp.120 - 150. Universidad de los Andes, Bogotá. 
2006b Plazas para los antepasados: descentralización y poder corporativo en las formaciones políticas preincaicas de los Andes Circumpuneños. Estudios Atacameños 31: 63 - 89.

2008 The Materiality of Ancestros: Chullpas and Social Memory in the Late Prehispanic History of the South Andes. En: Memory Work: Archaeologies of Material Practices (ed. por B. Mills y W.H. Walker), pp. 207 - 232. School of American Research Press, Santa Fe.

Nielsen, A. E. y L. Boschi

2007 Celebrando con los antepasados. Arqueología del espacio público en Los Amarillos, Quebrada de Humahuaca, Jujuy, Argentina. Mallku Ediciones, Buenos Aires.

Palma, J.

1997/98 Ceremonialismo mortuorio y registro arqueológico: apuntes sobre complejidad social. Relaciones XXII/XXIII: 179 - 202.

Paulotti, O., E. Molina y C. Visuara

1949 Contribución a la craneología de Catamarca Boletín Instituto Antropología 4: 249-268. Tucumán.

Politis, G.

2001 On archaeological praxis, gender bias and indigenous peoples in South America. Journal of Social Archaeology 1 (1): 90 - 107.

2006 El paisaje teórico y el desarrollo metodológico de la Arqueología en América Latina. Arqueología Suramericana 2 (2): 168 - 175.

Rodríguez Cuenca J.

2004 La antropología forense en la identificación humana. Universidad Nacional de Colombia.

Scattolin, M. C.

2006 Contornos y confines del universo iconográfico precalchaquí del valle de Santa María. Estudios Atacameños 32: 119 - 139.

Scattolin, M. C., M. F. Bugliani, L. Pereyra Domingorena y L. Cortes 2005 La Señora De Los Anillos, Una Tumba Presantamariana en el Valle de Yocavil. Intersecciones antropología 6: 29 - 41.

Seldes, V.

2004 Bioarqueología social. Nuevas perspectivas, nuevas preguntas de investigación. Estudios Sociales del NOA 7: 67 - 91. Instituto Interdisciplinario Tilcara. Facultad de Filosofía y Letras, Universidad de Buenos Aires.

2007 Aportes de la bioarqueología al estudio de la complejidad y desigualdad social en la Quebrada de Humahuaca (Jujuy, Argentina). Tesis de Doctorado. Facultad de Filosofía y Letras, Universidad de Buenos Aires.

2009 Mas allá de la adaptación. La interpretación del registro bioarqueológico. Anuario de Arqueología 1: 109-118. Escuela de Antropología - Facultad de Humanidades y Artes. Universidad Nacional de Rosario.

2010 Prácticas mortuorias en el noroeste argentino: una mirada a través del tiempo. El caso Omaguaca. IV Congreso Latinoamericano de Ciencias Sociais e Humanidades: Imagens da Morte. Universidad Salgado de Oliveira (PPGHIS - UNIVERSO), Niteroi, Brasil. 
Seldes, V. y M. F. Zigarán

2010 El Formativo en el Valle Calchaquí Norte. Una aproximación desde la Bioarqueología. Estudios Antropología - Historia, Nueva Serie, Número 1: 29 - 49.

Tarrago. M., M.A. Bordach y O. Mendonça

2005 El Cementerio de Rincon Chico 21, Santa María (Catamarca). Cuadernos FHyCS 29: 9-21. Universidad Nacional de Jujuy

\section{Vignati, M.}

1930 Los Cráneos trofeo de las sepulturas indígenas de la Quebrada de Humahuaca (Provincia de Jujuy). Archivos del Museo Etnográfico 1. Facultad de Filosofía y Letras. Universidad de Buenos Aires.

Williams, V. y B. Alberti

2006 Género y Etnicidad en la Arqueología Sudamericana. (compiladores). INCUAPA UNICEN. Buenos Aires

Wylie, A.

1997 The Engendering of Archaeology Refiguring Feminist Science Studies. Osiris 12: 80 99. 